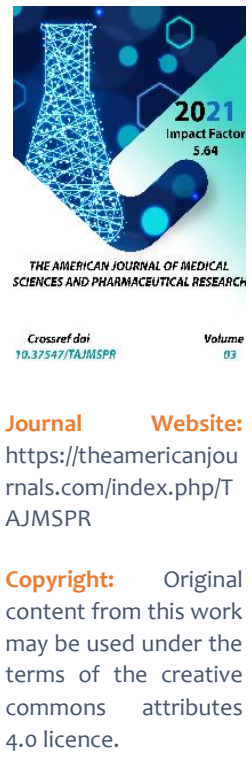

\title{
Role Of The PCSF Scale For Patients With Postcovid Syndrome
}

\section{Daminov B.T.}

Tashkent Pediatric Medical Institute, Tashkent, Republic Of Uzbekistan

Muminov D.K.

Tashkent Pediatric Medical Institute, Tashkent, Republic Of Uzbekistan

Davron Kadirovich Muminov

Department Off Faculty Internal Diseases, Occupatuonal Pathology, MFT, Hospital Internal Diseases And PID, Tashkent Pediatric Medical Institute, Tashkent, Republic Of Uzbekistan

\section{ABSTRACT}

Purpose of the study. Estimate the functional status of patients using the PCFS (Post-COVID-19 Functional Status) scale.

Material and research methods. There were 281 patients under observation, with the presence of clinical manifestations of post covid-19 syndrome. As a control group (CG), 20 patients who had undergone COVID-19 and whose disease ended in full recovery were examined. The assessment of the functional status of patients after suffering COVID-19 was carried out using the PCFS method - PostCOVID-19 Functional Status (https://osf.io/qgpdv/).

Research results. In the main group of patients, the average score of the questionnaire was $13.34 \pm$ 0.83 points. Patients who have identified a violation of their functional status at 1 point were 13 patients (4.63\%), at 2 points - 90 people (32.03\%), at 3 points - 117 people (41.64\%), at 4 points - 61 people. (21.71\%). In the control group, the patients assessed violations of their functional status at o points. The number of patients with extremely severe, severe and moderately severe COVID-19 was significantly higher in the main group than in the control group (30.61\% versus $20 \%, 35.94 \%$ versus $30 \%$, 33.45 versus $30 \%$, respectively) In the main group of patients, the average number of background diseases was significantly higher than in the control group $(p<0.05)$.

Conclusion. Patients with post covid syndrome have a higher score on the functional status scale (PCFS). Among the patients who scored 3 and 4 points on the PCFS test (significant limitation of daily activity due to symptoms associated with the previous infection and the need for help in self-care), there were more patients with a severe course of COVID-19 and background cardiovascular pathology and obesity. 


\section{KEYWORDS}

COVID-19, post covid syndrome, PCFS scale.

\section{INTRODUCTION}

Since the outbreak of the COVID-19 coronavirus pandemic, the focus has been on curbing the transmission of the SARS-COV-2 coronavirus, the development of severe acute respiratory syndrome, and on addressing the increasing number of critically ill patients in emergency departments [1]. To date, it has been unequivocally shown that COVID-19 can have a serious impact on the physical, cognitive, mental and social health status, including in patients with a mild form of the disease [2]. Articles appear in the literature describing the phenomenon of symptoms or various complaints that last more than 3-4 weeks and which are reported by patients who have had SARS-CoV-2 infection [3]. These conditions have gotten "long COVID" and "post covid syndrome". The term "post covid syndrome" has been defined to reflect interconnected or overlapping multisystems, fluctuating and often overlapping groups of symptoms with which patients seek medical attention $[3,4]$.

According to results of experts, given the heterogeneity of COVID-19 in terms of clinical and radiological manifestations, it is extremely important to have a simple tool to track the course of symptoms and their impact on the functional state of patients, that is, a scale that can measure the consequences of the disease beyond such outcomes. as mortality $[5,6]$. Objective: to determine the functional status of patients using the PCFS (Post-COVID-19 Functional Status) scale.

MATERIAL AND RESEARCH METHODS
There were 281 patients under observation, with the presence of clinical manifestations of postcoid syndrome. Inclusion criteria were: 1) virologically confirmed no earlier than 30 and later 7 days prior to inclusion in the COVID-19 study; 2) negative result of PCR research for SARS-CoV-19 at the time of inclusion in the study; 3) age 16-75 years. As a CG, 20 patients were examined who had undergone COVID-19 at the same time, in which the infection ended in full recovery (there are no pathological symptoms and signs that appeared in the acute period or after it and are not explained by other possible reasons). All patients of the main group and $\mathrm{FH}$ underwent examination, which included the collection of clinical and anamnestic data and the current state and course of the acute (infectious) period of COVID-19, laboratory and instrumental research methods.

The assessment of the functional status of patients after suffering COVID-19 was carried out using the PCFS method - Post-COVID-19 Functional Status (https://osf.io/qgpdv/). This method uses a questionnaire completed by the patient himself or a form filled out by the doctor in the process of interviewing the patient. In the case of both options, a point assessment of the functional status of patients who underwent COVID-19 was determined [6]. For the study, the questionnaire was translated into Russian and adapted for use in the Republic of Uzbekistan. The PCFS assesses the functional limitations of the quality of life, including lifestyle, sports, professional and social activities during the last week. 
Symptoms that limit functional status include shortness of breath, pain, weakness, fatigue, memory impairment, depression, and anxiety. In the case of applying 2 variants of assessment, the higher score is used. Statistical processing. All data obtained in the course of the study were entered into Excell summary tables. Intergroup comparison was performed using paired and unpaired Student's t test. In the case of nonparametric signs, the intergroup difference in the frequency distribution was assessed using the Chi-square table test and confirmation of its reliability according to the tables depending on the number of degrees of freedom. Results and discussion. A comparative assessment of the clinical characteristics of patients with COVID19 in the early rehabilitation period showed that the main group and the comparison group were comparable in age (49.48 \pm 12.54 and $49.10 \pm 12.61$ years, respectively). All patients included in the study underwent the PCFS test, which reflects the severity of the functional disorder of patients associated with a previous viral infection. In the main group of patients, the average score of the questionnaire was $13.34 \pm 0.83$ points. There were 13 patients with post covid syndrome who determined a violation of their functional status in 1 point (4.63\%), 2 points - 90 people (32.03\%), 3 points 117 people (41.64\%), 4 points - 61 people (21.71\%). The small number of patients who rated their condition disorders at 1 point is probably due to the method of recruiting patients - patients hospitalized due to unsatisfactory health were included. 1 point according to the PCFS method indicates a slight limitation of daily activity, which does not motivate the patient to go to a rehabilitation hospital. In almost a third of patients in the main group (90 people), the functional status was rated at 2 points, that is, a state that independently allows the main types of activity, but at the same time makes it possible to avoid certain types of activities or perform them intermittently, including due to pain, depression or anxiety. The largest number of patients (117 people) scored 3 points, which means restrictions on daily activity due to symptoms associated with the previous infection, but not requiring assistance in selfcare. 61 people rated their condition at 4 points, which indicates a significant limitation of the patient's daily activity due to symptoms associated with the previous infection and the patient needs help in self-care. In the CG, patients who underwent COVID-19 assessed violations of their functional status in connection with the infection at 0 points, which means that they have no restrictions on activity and complaints associated with the previous infection, including pain, anxiety, depression. It should be noted that when using the PCFS questionnaire, both forms were filled: forms for patients and forms for doctors. There was no significant difference in the points scored in both forms. The distribution of patients depending on the severity of COVID-19 was significantly different (Fig. 1) due to the absence of patients with a mild course of infection in the main group of patients ( $p$ $<0.001$ ). Thus, the number of patients with extremely severe, severe and moderately severe COVID-19 was significantly higher in the main group than in the CG (30.61\% versus $20 \%$, $35.94 \%$ versus $30 \%$, 33.45 versus $30 \%$, respectively). Moreover, the duration of the infectious period of the disease was comparable in both groups of patients. The severity of COVID-19 is also due to the large volume of interstitial pneumonia (Table 1). The volume of lung lesions according to MSCT data both in the infectious period and in the early rehabilitation period in patients with post covid 
syndrome was significantly greater than in patients in the comparison group ( $p<0.001)$. Accordingly, saturation at the time of inclusion in the study was significantly lower in patients of the main group ( $p<0.001)$. The study analyzed the comorbidity of patients in both groups. In the main group of patients, the average number of background diseases was significantly higher than in the CG $(4.46 \pm 2.18$ nosologies versus $3.45 \pm 1.88$ nosologies in the $C G, p<0.05)$. The groups did not differ in the frequency of occurrence of individual background pathologies, except for the cardiovascular system and obesity, which was significantly more frequent in the main group of patients $(p<0.05)$. The analysis of the obtained results showed that in the main group, among the patients who scored 3 and 4 points on the PCFS test, there were more patients with a severe course of COVID-19 and background pathology of cardiovascular and obesity.

Table 1.

Clinical characteristics of patients included in the study

\begin{tabular}{|c|c|c|}
\hline & $\begin{array}{c}\text { Main group } \\
(\mathrm{n}=281)\end{array}$ & $\begin{array}{c}\text { Comparison group } \\
(\mathrm{n}=20)\end{array}$ \\
\hline Age & $49,48 \pm 12,54$ & $49,10 \pm 12,61$ \\
\hline $\begin{array}{c}\text { duration of the disease, days } \\
\text { Lung lesion volume according to MSCT data, } \\
\text { infectious period,\% }\end{array}$ & $13,34 \pm 4,55$ & $11,80 \pm 3,74$ \\
\hline $\begin{array}{c}\text { Lung lesion volume according to MSCT data, early } \\
\text { rehabilitation period,\% }\end{array}$ & $44,47 \pm 23,97$ & $23,00 \pm 19,63 * * *$ \\
\hline Saturation, \% & $92,67 \pm 5,03$ & $11,25 \pm 10,50 * * *$ \\
\hline
\end{tabular}

Note: * reliability of differences between groups: two signs $-p<0.01$, three signs $-p<0.001$. 
Figure 1.

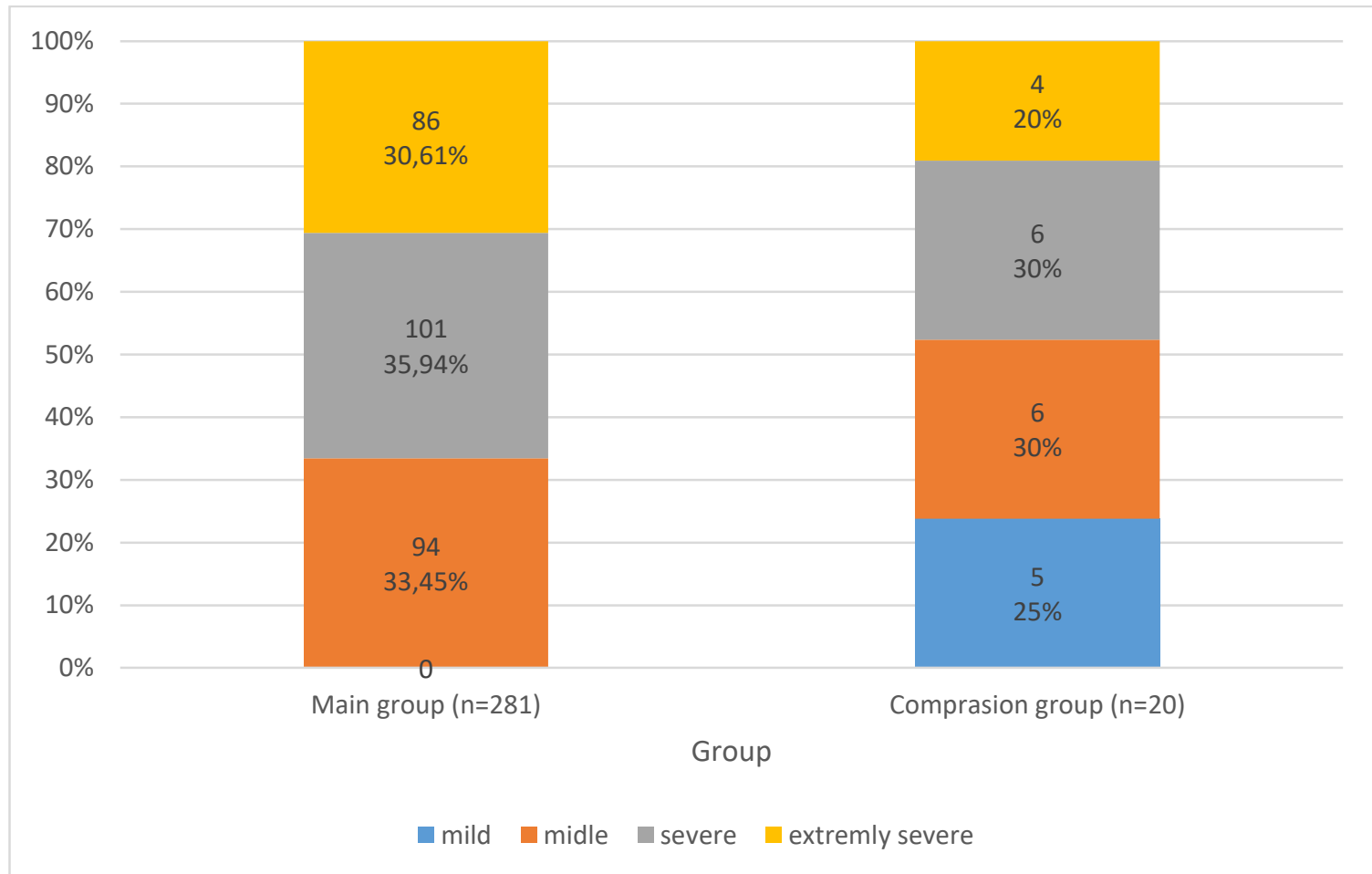

Frequency of various degrees of severity of COVID-19 in groups of patients examined in the early rehabilitation period $(\mathrm{chi}$ square $=68.22, \mathrm{p}<0.001$ )

International experts believe that with the sheer number of COVID-19 patients requiring follow-up, a simple and reproducible tool will help guide the prudent use of medical resources. On February 18, 2020, the World Health Organization proposed an "Order Scale of Clinical Improvement" [7] with categories mainly based on the type of treatment that will be used as the primary endpoint in acute phase trials. However, because this scale is focused on inpatient care, it reflects the long-term outcomes of COVID-19 and post-discharge treatment. Boon GJAM et al. [8] proposed an ordinal scale for assessing a patient's functional limitations after an episode of venous thromboembolism (PVFS). This PVFS scale (after minor adaptation) has been found useful in COVID-19 given that critically ill COVID19 patients have a high incidence of pulmonary embolism itself, along with myocardial damage I myocarditis and neurological complications $[9,10]$. Studies involving 1939 people with symptoms associated with coronavirus infection, approximately three months after illness, demonstrated the construct validity of the PCFS. The authors note that the main advantage of the scale is its ease of use and suggest using it when referring to expert (outpatient) clinics or rehabilitation programs [11]. Thus, the study found that patients who have post covid syndrome in the early rehabilitation period after suffering COVID-19 
have a higher score on the functional status scale (PCFS). Among the patients who scored 3 and 4 points on the PCFS test (significant limitation of daily activity due to symptoms associated with the previous infection and the need for help in self-care), there were more patients with a severe course of COVID-19 and underlying cardiovascular pathology and obesity.

\section{REFERENCES}

1. Johns Hopkins University Coronavirus Resource Center. https://coronavirus.jhu.edu/ Date last accessed: April 2020.

2. Simpson R, Robinson L. Rehabilitation after critical illness in people with COVID-19 infection. Am J Phys Med Rehabil 2020; 99: 470-474. doi:10.1097/PHM.000000000000144 3.

3. Mandal S., Barnett J., Brill S.E. $и$ соавт.; ARC Study Group. 'LongCOVID': a cross-sectional study of persisting symptoms, biomarker and imaging abnormalities following hospitalisation for COVID-19. Thorax, 2020. doi: 10.1136/thoraxjnl-2020-215 8180.

4. NICE. Rehabilitation after critical illness. NICE Clinical Guideline 83. London: National Institute for Health and Care Excellence; 2009 (http://www.nice.org.uk/guidance/cg 83, accessed 21 May 2020).

5. Simpson R, Robinson L. Rehabilitation after critical illness in people with COVID-19 infection. Am J Phys Med Rehabil 2020; 99: 470-474. doi:10.1097/PHM.000000000000144 3.
6. Klok, F. A., Boon, G., Barco, S., Endres, M., Geelhoed, J., Knauss, S., Rezek, S. A., Spruit, M. A., Vehreschild, J., \& Siegerink, B. (2020). The Post-COVID19 Functional Status scale: a tool to measure functional status over time after COVID-19. The European respiratory journal, 56(1), 2001494. https://doi.org/10.1183/13993003.0149 4-2020.

7. World Health Organization www.who.int/blueprint/prioritydiseases/key-action/COVID-19 Treatment Trial Design Master Protocol synopsis Final 18022020.pdf WHO R\&D Blueprint. Novel Coronavirus: COVID-19 Therapeutic Trial Synopsis. Draft February 18, 2020.

8. Boon GJAM, Barco S, Bertoletti L, et al. . Measuring functional limitations after venous thromboembolism: optimization of the post-VTE functional status (PVFS) scale. Thromb Res 2020; 190: 45-51. doi:10.1016/j.thromres.2020.03.020.

9. Lodigiani C, lapichino G, Carenzo L, et al. . Venous and arterial thromboembolic complications in COVID-19 patients admitted to an academic hospital in Milan, Italy. Thromb Res 2020; 191: 9-14. doi:10.1016/j.thromres.2020.04.024.

10. Klok FA, Kruip MJHA, van der Meer NJM, et al. Incidence of thrombotic complications in critically ill ICU patients with COVID-19. Thromb Res 2020; 191: 145-147. doi:10.1016/j.thromres.2020.04.013.

11. Machado, F.V.C., Meys, R., Delbressine, J.M. et al. Construct validity of the Post-COVID-19 
The American Journal of Medical Sciences and Pharmaceutical Research

(ISSN - 2689-1026)

Published: July 30, 2021 | Pages: 92-98

Doi: https://doi.org/10.37547/TAJMSPR/Volume03Issue07-07

IMPACT FACTOR

2021: 5.64

OCLC - 1121105510

Functional Status Scale in adult subjects with COVID-19. Health Qual

Life Outcomes 19, 40 (2021).

https://doi.org/10.1186/s12955-021-

01691-2. 